OPEN ACCESS

Edited by:

Lu Chen,

Tianjin University of Traditional Chinese

Medicine, China

Reviewed by:

Md Sanaullah Sajib,

United States Food and Drug

Administration, United States

Pinaki Misra

Mayo Clinic, United States

${ }^{*}$ Correspondence:

Qian Ba

qba@shsmu.edu.cn

Hui Wang

huiwang@shsmu.edu.cn

Jingquan Li

jqli@shsmu.edu.cn

Specialty section:

This article was submitted to

Pharmacology of Anti-Cancer Drugs,

a section of the journal

Frontiers in Oncology

Received: 25 July 2021

Accepted: 22 September 2021

Published: 14 October 2021

Citation:

Chen F, Li J, Wang H and Ba Q (2021)

Anti-Tumor Effects of Chinese

Medicine Compounds by Regulating Immune Cells in Microenvironment.

Front. Oncol. 11:746917.

doi: 10.3389/fonc.2021.746917

\section{Anti-Tumor Effects of Chinese Medicine Compounds by Regulating Immune Cells in Microenvironment}

\author{
Fengqian Chen, Jingquan $\mathrm{Li}^{*}$, Hui Wang ${ }^{*}$ and Qian $\mathrm{Ba}^{*}$ \\ State Key Laboratory of Oncogenes and Related Genes, Center for Single-Cell Omics, School of Public Health, Shanghai \\ Jiao Tong University School of Medicine, Shanghai, China
}

As the main cause of death in the world, cancer is one of the major health threats for humans. In recent years, traditional Chinese medicine has gained great attention in oncology due to the features of multi-targets, multi-pathways, and slight side effects. Moreover, lots of traditional Chinese medicine can exert immunomodulatory effects in vivo. In the tumor microenvironment, tumor cells, immune cells as well as other stromal cells often coexist. With the development of cancer, tumor cells proliferate uncontrollably, metastasize aggressively, and modulate the proportion and status of immune cells to debilitate the antitumor immunity. Reversal of immunosuppressive tumor microenvironment plays an essential role in cancer prevention and therapy. Immunotherapy has become the most promising strategy for cancer therapy. Chinese medicine compounds can stimulate the activation and function of immune cells, such as promoting the maturation of dendritic cells and inducing the differentiation of myeloid-derived suppressor cells to dendritic cells and macrophages. In the present review, we summarize and discuss the effects of Chinese medicine compounds on immune cells in the tumor microenvironment, including innate immune cells (dendritic cells, natural killer cells, macrophages, and myeloid-derived suppressor cells) and adaptive immune cells $\left(\mathrm{CD} 4^{+} / \mathrm{CD} 8^{+} \mathrm{T}\right.$ lymphocytes and regulatory $T$ cells), and the various immunomodulatory roles of Chinese medicine compounds in cancer therapy such as improving tumor-derived inflammation, enhancing the immunity after surgery or chemotherapy, blocking the immune checkpoints, et al., aiming to provide more thoughts for the anti-tumor mechanisms and applications of Chinese medicine compounds in terms of tumor immunity.

Keywords: Chinese medicine compounds, cancer, immune cell, antitumor immunity, microenvironment

\section{INTRODUCTION}

Due to high morbidity and mortality, the malignant tumor has long been a hot issue in society. Surgery and chemotherapy are the primary treatments for cancers. Over the past decades, although researchers have devoted themselves to exploiting and exploring more effective treatments for cancers, the morbidity and mortality of malignant tumors are still high all over the world (1). Due to the heterogeneity and complexity of tumors, the cancer treatment focus from single target to multiple targets (2), and the tumor environment (TME) has attracted much attention. There, tumor-infiltrating 
immune cells play pivotal roles in tumor immunity and related functions. Chinese medicine compounds refer to the prescription composed of multiple kinds of traditional Chinese medicine (TCM) or the main components of TCM, which are used for treating malignant tumors given the characteristics of multipathways, multi-targets, slight side effects, and immunity enhancement (3). More and more Chinese medicine compounds have been recognized for their effects on tumor immunity, such as regulating the proliferation and activation of immune cells and their cytokines $(4,5)$. However, the impacts and mechanisms of Chinese medicine compounds on distinct immune cells and anticancer immunity are diverse and complicated. Herein, we provide an overview of the anti-cancer mechanisms of Chinese medicine compounds by regulating the diverse immune cells (Figure 1) and various underlying immunomodulatory pathways in cancer therapy (Figure 2).

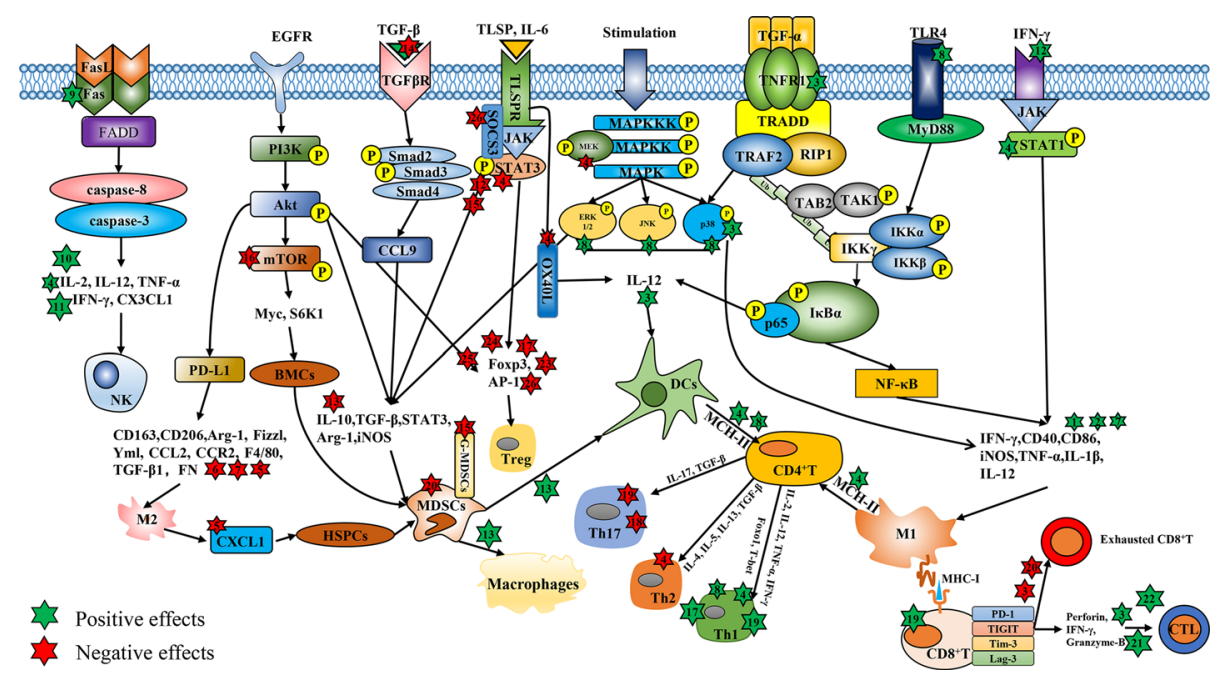

FIGURE 1 | The regulatory effects of Chinese medicine compounds in immune cells. Chinese medicine compounds: 1. Modified Si-Jun-Zi Decoction; 2. Haimufang decoction; 3. Compound kushen injection; 4. Yu-ping-feng; 5. XIAOPI formula; 6. Dahuang Zhechong Pills; 7. Jianpi Yangzheng; 8. Yangyin Wenyang; 9. Jinfukang; 10. Tien-Hsien liquid; 11. ACNO; 12. Yanghe Decoction; 13. Jianpi Huayu Decoction; 14. Baoyuan Jiedu decoction; 15. Ze-Qi-Tang; 16. Shuangshen granules; 17. Quxie capsule; 18. Compound Sophorae Decoction; 19. JC-001 20. Shugan Jianpi formula; 21. Xiao-Ai-Ping; 22. Shenqi Fuzheng Injection; 23. Feiyanning Decoction; 24. Yi-Yi-Fu-Zi-Bai-Jiang-San; 25. Xihuang Pill; 26. Fuzheng Fangai.

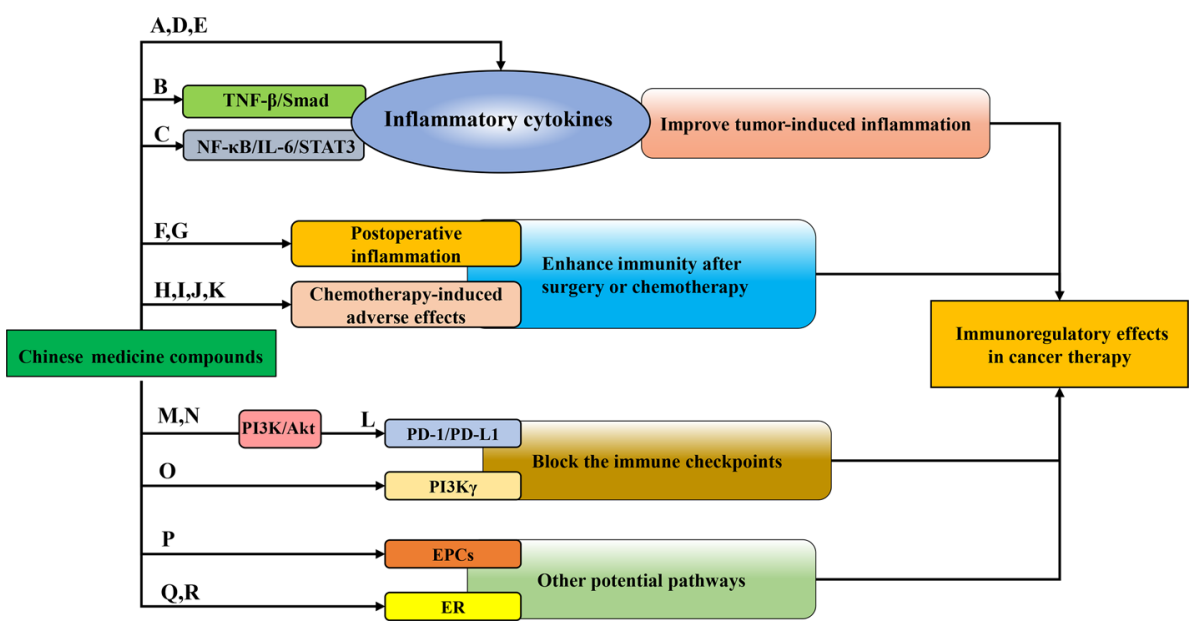

FIGURE 2 | The immunoregulatory patterns of Chinese medicine compounds in cancer therapy. Chinese medicine compounds: (A) Banxia Xiexin decoction; (B) Compound kushen injection; (C) NHE-06; (D) Ruyiping+Platycodon grandiflorum; (E) Kejinyan decoction; (F) DahuangZhechong Pills; (G) San Huang decoction; (H) PHY906; (I) Ciji-Hua'ai-Baosheng; (J) Gegen Qinlian decoction; (K) Yangyin Fuzheng Decoction; (L) Bu Fei Decoction; (M) Huoxue Yiqi Recipe-2; (N) Bu-zhongyi-qi decoction; (O) Modified Jian-pi-yang-zheng decoction; (P) Danggui Buxue decoction; (Q) XH formula; (R) Shugan Liangxue decoction. 


\section{IMMUNE CELLS IN TUMOR MICROENVIRONMENT}

TME changes with tumor development and represents diverse immune cell composition and characteristics. Recently, immunotherapy becomes the most promising therapeutic strategy for cancer. In the TME, the functions of diverse immune cell populations function beneficially or harmfully through the local direct/indirect interaction with cancer cells. Typical immune cells in the TME include macrophages, dendritic cells (DCs), natural killer cells (NK cells), and myeloid-derived suppressor cells (MDSCs) in the innate immune system, as well as $\mathrm{CD}^{+} / \mathrm{CD}^{+} \mathrm{T}$ lymphocytes and regulatory $\mathrm{T}$ cells (Tregs) in the adaptive immune system. As the first defense line against pathogens, macrophages serve as typical antigen-presenting cells (APCs) with the functional capability of phagocytosis and antigen presentation. Tumorassociated macrophages (TAMs), which are recruited into tumors from monocytes in peripheral blood or macrophages in normal tissues, have been proven to assist tumor growth, angiogenesis, and metastasis, thus are considered as a promoter for tumor progression (6). In the TME, TAMs undergo differentiation with distinct properties in response to different stimuli, mainly M1 and M2 phenotypes. M2 macrophages that are activated by alternative pathways play a leading role in TAMs, which are capable of inducing cancer initiation and angiogenesis. Conversely, M1 macrophages are activated classically and have a strong ability of phagocytosis, antigen presentation, and secretion of various pro-inflammatory cytokines such as interleukin-6 (IL-6), IL-12, IL-23, tumor necrosis factor- $\alpha$ (TNF- $\alpha$ ) and chemokines to effectively eliminate pathogens or tumor cells in the body (7). DCs are the strongest APCs in human immunity, which enable to efficiently ingest, process, and present antigens to naïve $\mathrm{T}$ lymphocytes to initiate adaptive immune responses, acting as a vital bridge linking innate immunity and adaptive immunity (8). Whereas, tumor-infiltrating DCs generally appear to be immature and functional paralysis, thereby failing to initiate or modulate adaptive immunity and gradually leading to immunosuppression (9). NK cells are the cytotoxic lymphocytes of the innate immune system. Although lack antigen receptors, they are regarded as the key cancer defensor due to the cytotoxic ability to quickly kill nearby target cells without antigen in advance. Similarly, there are a variety of endogenous mechanisms to resist NK cells from attack and deep infiltration, leading to the failed cytotoxicity (10). Moreover, MDSCs refer to a heterogeneous population of cells consisting of early myeloprogenitor cells, naïve granulocytes, as well as immature macrophages and DCs with various differentiation degrees, which display strong T-cell suppression function. Proinflammatory cytokines could induce the generation of MDSCs in tumor microenvironment and defect the differentiation of MDSCs into mature immune cells. Over the past years, numerous studies have shown that MDSCs could promote cancer progression in terms of cancer cell proliferation and invasion, tumor angiogenesis, and drug resistance (11).
As immunotherapy has been widely studied, $\mathrm{CD} 4^{+}$ $\mathrm{T}$ lymphocytes, also known as helper $\mathrm{T}$ cells (Th), have attached much attention due to the remarkable effects on the adaptive immune system. The naive $\mathrm{T}$ lymphocytes (Th0) differentiate into different subsets, such as Th1, Th2 and Th17. The Th1 immune response can be usually inhibited in a variety of malignant tumors, which manifests as the down-regulation of TNF- $\alpha$, IL-2, IFN- $\gamma$ and IL-2, and defecting the proliferation of cytotoxic T cells, macrophages and NK cells. On the contrary, Th2-biased immune response is up-regulated in cancer progression (12). The cytokines IL-4, IL-5 and IL-13 secreted by Th2 cells have been found to facilitate tumor growth (13). In addition, the ratio of Th1/Th2 refers to a crucial factor to maintain the normal immune state, and the imbalance of Th1/ Th2 often occurs in immune-related diseases including cancer. Th17 is another helper $\mathrm{T}$ cell, which was named from the ability to secrete the pro-inflammatory cytokine IL-17. Th17 has been considered as a potential target for tumor treatment, however, in different tumors, Th17 showed inconsistent effects $(14,15)$. The subset of $\mathrm{CD}^{+} \mathrm{T}$ lymphocytes, also called cytotoxic $\mathrm{T}$ cells (CTLs), is an important guardian of the adaptive immune system. CTLs exert great cytotoxic effects on tumor cells, in other words, they perform cellular immunity by lysing or inducing apoptosis of targeted cells. However, with the infiltration of $\mathrm{CD}^{+} \mathrm{T}$ lymphocytes into tumor tissues, they undergo exhaustion in form of suppressed proliferation and even apoptosis (16). Tregs are a unique subset of $\mathrm{CD} 4^{+} \mathrm{CD} 25^{+}$ $\mathrm{T}$ lymphocytes and are augmented in various cancers. Tregs in the TME serve as a potent immunosuppressive factor and resist anti-tumor immunity (17). Additionally, the balance of Th17/ Treg is likely to be disturbed by TAMs in the TME (18). Therefore, it is a feasible immunotherapeutic strategy to reshape TME by modulating the activity and function of immune cells.

\section{THE REGULATORY EFFECTS OF CHINESE MEDICINE COMPOUNDS ON INNATE IMMUNE CELLS}

\section{Macrophages}

Targeted consumption or regulation of TAMs has become a potential cancer treatment strategy (19), such as activating TAMs towards M1-type polarization, suppressing M2-type polarization, and inducing M2 polarizing to M1. Numerous Chinese medicine compounds have been reported to exert inhibitory effects on tumor growth and metastasis through targeting macrophages (Figure 1). Th1 cytokines IFN $-\gamma$ are conducive to M1 activation in the TME (20). Modified Si-Jun$Z i$ Decoction stimulated the secretion of GM-CSF, IFN- $\gamma$, IL- $1 \alpha$ and IL-3, increased the number of macrophages, thus activating the innate immune system to remove colorectal cancer (CRC) cells (21). The elevated expression of phenotypic marker CD86 and CD40 stand for the activation of M1 macrophages to some extent. Ma et al. observed that Haimufang (HMF) decoction 
enhanced the generation of nitric oxide (NO) and reactive oxygen species (ROS), the secretion of cytokines TNF- $\alpha$, IL- $1 \beta$, IL-12 p70, and IL-6, and the expression of M1 marker CD40 and CD86, with time- and dose-dependent manners in RAW264.7 cells, but had no discernible impact on M2 phenotypic marker CD206. As a result, HMF promoted the M1 polarization and enhanced the phagocytosis ability (22). In hepatocellular carcinoma (HCC), Yang et al. reported that Compound kushen injection (CKI) facilitated the activation of macrophages by stimulating TNF receptor superfamily member 1 (TNFR1)-mediated NF- $\kappa \mathrm{B}$ and p38 MAPK signaling pathways, thereby accelerating M1 polarization and alleviating TAMs-mediated immunosuppression (23). Yu-pingfeng (YPF) has usually been prescribed to enhance human immunity, which was reported to up-regulate the secretion of IL-1 $\beta$ and IL-12, the level of iNOS as well as the phosphorylation and activation of signal transducers and activators of transcription 1 (STAT1) in RAW264.7 cells to propel the M1 polarization and enhance $\mathrm{M} 1$-induced $\mathrm{CD} 4{ }^{+} \mathrm{T}$ cell activation and Lewis lung cancer (LLC) lysis (24). Furthermore, Chinese medicine compounds could restrain the function of M2 and facilitate the conversion of M2 to M1. XIAOPI (XP) formula remarkably prevented the promoting effect of TAMs on the proliferation and self-renewal activity of breast cancer cell lines (MDA-MB-231 and 4T1) in a co-culture system, and reversed the TAMs-mediated C-X-C Motif Chemokine Ligand 1 (CXCL1) secretion and $\beta$-catenin signal, thus attenuating the self-renewal activity and chemotherapy resistance of breast cancer stem cells (CSCs). Moreover, XP weakened the polarization of M2 by down-regulating the expression of CD163, CD206 and Arg1 as well as the transcriptional activity of CXCL1 in a dose-dependent manner (25). C-C Motif Chemokine Ligand-2 (CCL2) refers to an important chemokine for monocyte/macrophage chemotaxis (26). Chen et al. reported that CCL2 boosted the number of macrophages in the liver and induced M2 polarization, which could be prevented by Dahuang Zhechong Pills (DZP) through the suppressed secretion of CCL2 and its receptor CCR2, along with the low level of F4/80, TGF- $\beta 1$ and FN to attenuate hepatic fibrotic status. DZP also reversed tumormediated M2 polarization of macrophages to reshape the immunosuppressive TME and inhibit the liver metastasis of CRC (27). Jianpi Yangzheng (JPYZ), the ingredients for invigorating qi and spleen of Jianpi Yangzheng Xiaozheng decoction, can promote the transformation of M2 to M1 with the elevation of CD86 and reduction of CD206 and CD163. The mRNA levels of M1-related genes (IL-1 $\beta$, IL-12 and TNF- $\alpha$ ) were enhanced by JPYZ while those of M2-related genes (Arg-1, Fizzl and Yml) were attenuated (28). Consequently, Chinese medicine compounds indeed regulate the activation and differentiation of TAMs to exert anti-tumor activity (Table 1).

\section{Dendritic Cells (DCs)}

Chinese medicine compounds could enhance the maturation and antigen presentation ability of DCs to induce the activation of naïve T lymphocytes and participate in the regulation of $\mathrm{T}$ cell differentiation (Table 1). IL-12 is closely involved in the antigen presentation function of DCs (41). Zhao et al. optimized a new traditional Chinese medicine formula Yangyin Wenyang (YYWY) on the basis of Jinfukang. YYWY intervention drove the impregnation of DCs, $\mathrm{CD} 4^{+}$and $\mathrm{CD}^{+}{ }^{+} \mathrm{T}$ cells in LLC tissues, and raised the mRNA levels of IFN- $\gamma$, IL-1 $\beta$, TNF- $\alpha$ and IL-12. In addition, YYWY stimulated MAPK and NF- $\kappa B$ signaling pathways in bone marrow dendritic cells (BMDCs) to accelerate the maturation of DCs and the proliferation and differentiation of $\mathrm{T}$ cells together (29). TSLP, an immunerelated factor, can activate DCs and involve in $\mathrm{T}$ cell differentiation, which raises the expression of Th2-polarizing molecule CD252 (OX40L), and induces Th2 response (42). Yao et al. reported that YPF reduced the expression of TSLP and OX40L to induce the maturation of DCs and the expression of CD80, CD86, MHC-II as well as IL-12 (12). Furthermore, DCs combine with tumor cells to form fusion cells (FCs), which play an inhibitory role in tumor development. Yang et al. found that fusion cells of dendritic colon cancer stem cells (DC-cCSC FCs) facilitated the proliferation of cytokines induce killer (CIK) cells. The cytotoxicity of DC-cCSC FCs and CIK cells against cCSCs was further improved by IL-12 and could be enhanced by the Chinese medicine CKI (30). In summary, Chinese medicine compounds are capable to activate DCs. Although some studies have proven that Chinese medicine compounds elevate the function of NK cells in the TME by promoting the secretion of IL-12 (Figure 1), more researches about the mechanisms are demanded.

\section{Natural Killer Cells (NK Cells)}

Recently, the anti-tumor activity of exogenously activated and amplified NK cells has been proved in clinical (43). Some studies have shown that activating NK cells may be an effective approach for Chinese medicine compounds to suppress the advance of tumors (Table 1). For example, Luo et al. confirmed that YPF propelled the infiltration, proliferation, and killing activity of NK cells in tumor tissues to prolong the survival of LLC tumorbearing mice. Elimination of NK cells could remarkably reverse the inhibitory effect of YPF on lung cancer, indicating that NK cells were the potential target cells for YPF-mediated lung cancer treatment (31). Additionally, Que et al. found that Jinfukang was conducive to the secretion of C-X3-C Motif Chemokine Ligand 1 (CX3CL1) in circulating tumor cells (CTCs) and the recruitment of CTCs to NK cells. Meanwhile, Jinfukang significantly accelerated NK cells-mediated CTCs apoptosis and prevented lung cancer metastasis through activating Fas/FasL signaling pathway, manifested as the up-regulated FasL and secretion of TNF- $\alpha$ in NK cells (32). In addition, Tien-Hsien liquid (THL) accelerated the secretion of immune factors including IFN- $\gamma$, IL-2 and TNF- $\alpha$ to augment the cytotoxicity of NK cells and CTLs and mitigate the tumor development (33). Finally, Li et al. reported a new traditional Chinese medicine compound anti-cancer No.1 (ACNO) which could dose-dependently enhance the cytotoxicity of NK cells by up-regulating the secretion of IL-2, IL-12, and INF- $\gamma$ (34) (Figure 1). In brief, Chinese medicine compounds can interfere with tumor growth by the way of enhancing the killing activity of NK cells. 
TABLE 1 | Innate immune cells impacted by Chinese medicine compounds.

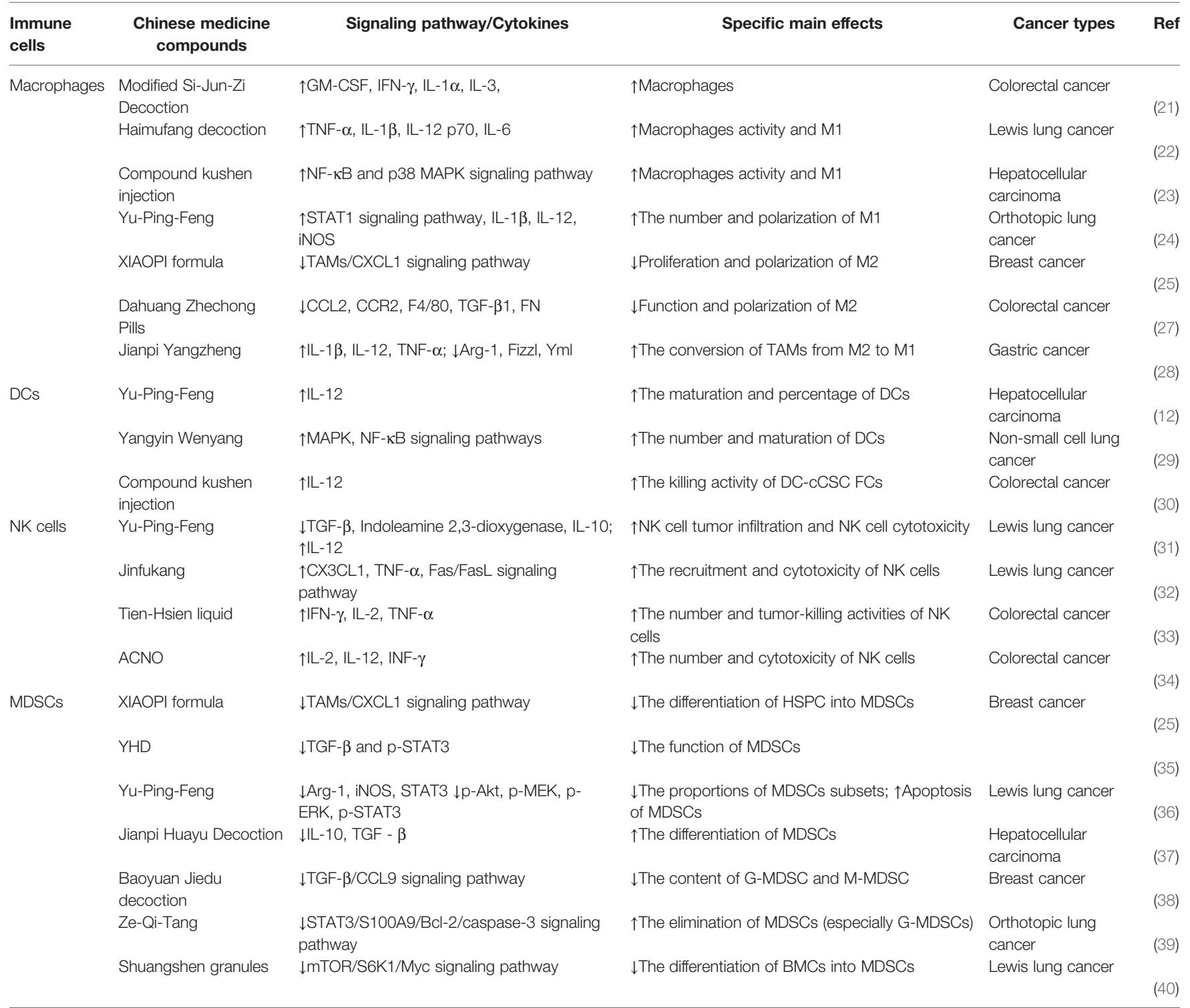

$\uparrow$, Up-regulated or enhanced; $\downarrow$, Down-regulated or inhibited.

\section{Myeloid-Derived Suppressor Cells (MDSCs)}

Researches have suggested that Chinese medicine compounds abated the immunosuppression in the TME by facilitating the apoptosis and differentiation of MDSCs to enhance immune response (Table 1). Mao et al. observed that Yanghe Decoction (YHD) had immunomodulatory effects on 4T1 breast tumors, not only boosting the amount of NKT and $\mathrm{CD} 4^{+} \mathrm{T}$ cells as well as the secretion of IFN- $\gamma$ and p-STAT1, but also attenuating the recruitment of MDSCs through JAK/STAT3 signaling pathway (35). YPF suppressed the mRNA levels of immunosuppressive genes in MDSCs, including Arg-1, iNOS and STAT3, and decreased the levels of p-Akt, p-MEK, p-ERK and p-STAT3 to prevent MDSCs augment and induce their apoptosis, which further modulated the proportion of $\mathrm{T}$ cell subsets by increasing $\mathrm{CD}^{+} / \mathrm{CD}^{+} \mathrm{T}$ lymphocytes and decreasing Treg proportion, thus reshaping the immune TME and preventing the lung cancer progression (36). Moreover, Xie et al. proved that Jianpi Huayu Decoction (JHD) attenuated the expression of IL-10 and TGF- $\beta$ in tumor tissues and accelerated the differentiation of MDSCs into macrophages and DCs. JHD decreased the content of ROS in MDSCs, inhibited CD4 ${ }^{+}$ $\mathrm{T}$ cells proliferation and the percentages of Th17 and Treg, but elevated the proportion of CTLs and DCs to relieve the MDSCsmediated immunosuppressive state in HCC (37). Another research reported that Baoyuan Jiedu decoction (BYJD), a traditional Chinese medicine formula composed of Astragalus, Ginseng, Aconite root, Honeysuckle, Angelica, and Licorice, inhibited the TGF- $\beta$ /CCL9 signaling pathway and reduced the number of MDSCs in peripheral blood and spleen of breast 
cancer (4T1)-bearing mice. Furthermore, BYJD blocked the recruitment of MDSCs in lung, the metastatic organ of breast cancer, thereby improving the pre-metastatic niche (PMN) and prolonging the survival of tumor-bearing mice (38). Xu et al. found that the medium dose of Ze-Qi-Tang (ZQT) had the strongest anti-tumor activity. In lung carcinoma in situ, ZQT could eliminate MDSCs in a dose-dependent manner, reduce the recruitment of MDSCs, and enhance the infiltration of $\mathrm{T}$ cells to reverse MDSCs-mediated immunosuppression. Thoroughly discovered that ZQT suppressed the level of STAT3, p-STAT3 and anti-apoptotic protein $\mathrm{Bcl}-2$, increased the expression of pro-apoptotic protein Bax, cleaved caspase- 3 and PARP to induce the apoptosis of G-MDSCs in lung carcinoma in situ. However, after G-MDSCs were completely depleted, the cytotoxicity of $\mathrm{CD}^{+} \mathrm{T}$ cells and the inhibitory effect of MDSCs had no significant difference between ZQT group and the control group, suggesting that the anti-tumor activity of ZQT was achieved by targeting G-MDSCs subset (39). Chinese medicine compounds also could decline the generation of MDSCs. Shuangshen granules restrained the expression of mTOR, S6K1 and Myc to block the differentiation of bone marrow cells (BMCs) into MDSCs in a dose-dependent manner, thus preventing lung metastasis (40). XP impaired the activation of hematopoietic stem-progenitor cells (HSPCs) as well as the differentiation of HSPCs into MDSCs through TAMs/ CXCL1 signaling pathway to inhibit lung metastasis of breast cancer (25). Therefore, it may be a promising therapeutic way for Chinese medicine compounds to improve TME through targeting MDSCs (Figure 1).

\section{THE REGULATORY EFFECTS OF CHINESE MEDICINE COMPOUNDS ON ADAPTIVE IMMUNE CELLS}

\section{CD4 $^{+}$T Lymphocytes}

Some Chinese medicine compounds are capable of exerting antitumor effects through facilitating $\mathrm{CD}^{+} \mathrm{T}$ lymphocytes differentiate to Th1 and attenuating Th2 response (Table 2). The activation and differentiation of $\mathrm{CD} 4^{+} \mathrm{T}$ lymphocytes tend to be affected by APCs, such as macrophages and DCs. YPF enhanced the antigen presentation ability of macrophages by up-regulating the level of MHC II, thereby activating the LLC cell lysis mediated by $\mathrm{CD}^{+}{ }^{+} \mathrm{T}$ cells. Meanwhile, YPF enhanced the secretion of Th1 cytokines (IL-2, IL-12) but repressed the level of Th2 cytokines (TGF- $\beta$, IL-4) (24). In addition, in H-22 tumor-bearing mice, YPF elevated the ratio of Th1/Th2 (IFN- $\gamma$ / IL-4) through mature DCs, thus ameliorating Th2-biased immune state (12). Similarly, YYWY exerted the anti-tumor effect by inducing DCs mature, which subsequently activated $\mathrm{CD}^{+}{ }^{+} \mathrm{T}$ lymphocytes and enhanced Th1 function (29). Chen et al. found that in CT26 tumor-bearing mice, Quxie capsule (QX) intervention boosted the expression of Foxol and T-bet, along with the augment of Th1 and Th1/Th2, to propel the immune response in TME (44). Th17 has been considered as a potential target for tumor treatment in several studies. Deng et al. confirmed that Th17 was conducive to the occurrence of Ulcerative Colitis-Related Colorectal Cancer (UCRCC), however, Compound Sophorae Decoction (CSD) remarkably reversed the increasing number of Th17 and the secretion of IL-17 to relieve inflammation in UCRCC (45). Chuang et al. reported that in the co-culture system of spleen cells and LLC cells, JC-001 enhanced Th1 function and suppressed Th17 function by abating the secretion of TGF- $\beta$ and IL-17A to prevent tumor progression (46). In liver cancer, JC-001 had the same effects on Th1 and Th17 function, thereby alleviating Treg-mediated immunosuppression (47). Taken together, Chinese medicine compounds control tumor growth by modulating the differentiation of $\mathrm{CD}^{+} \mathrm{T}$ cells, enhancing Th1 immune response as well as restraining the function of Th2 and Th17 (Figure 1).

\section{$\mathrm{CD}^{+}{ }^{\mathrm{T}}$ Lymphocytes}

Restoring $\mathrm{CD}^{+} \mathrm{T}$ lymphocytes function has always been regarded as an effective method to facilitate tumor immunity (16). Some studies have demonstrated that Chinese medicine compounds lead to the augment of $\mathrm{CD}^{+} \mathrm{T}$ lymphocytes in tumor tissues $(29,33,36)$. In addition, Chinese medicine compounds bring vital impacts on the activity and exhaustion of $\mathrm{CD}^{+} \mathrm{T}$ cells (Table 2). CKI combined with sorafenib dramatically suppressed function-inhibitory receptors in $\mathrm{CD}^{+}$ $\mathrm{T}$ cells, including lymphocyte-activation gene 3 (Lag-3), programmed cell death protein 1 (PD-1), T-cell immunoreceptor with Ig and ITIM domains (TIGIT), and Tcell immunoglobulin and mucin-domain containing-3 (Tim-3) to reduce the depletion of $\mathrm{CD}^{+} \mathrm{T}$ cell. At the same time, the combination therapy strengthened the cytotoxicity of $\mathrm{CD}^{+} \mathrm{T}$ cells by accelerating the production of TNF- $\alpha$, Perforin, IFN- $\gamma$ and Granzyme-B. When $\mathrm{CD} 8^{+} \mathrm{T}$ lymphocytes were blocked, the anti-tumor activity of combination therapy disappeared, suggesting that $\mathrm{CD}^{+} \mathrm{T}$ cells were necessary for CKI-mediated HCC treatment (23). Lu et al. reported that, in depression breast cancer mice, Shugan Jianpi formula combined with chemotherapy drug gemcitabine (GEM) effectively repressed the apoptosis of $\mathrm{CD}^{+} \mathrm{T}$ cells and significantly reduced MDSCs to enhance the immune surveillance, thereby preventing the progress of breast cancer (48). It has been shown that Xiao-Ai-Ping, an adjuvant injection for tumor therapy, propelled the infiltration and function of $\mathrm{CD}^{+} \mathrm{T}$ cells in the TME of LLC, mainly manifested as the increase in Perforin, IFN- $\gamma$ and Granzyme-B (49). At last, JC-001 drove the generation of $\mathrm{CD}^{+} \mathrm{T}$ cells in LLC1 tumor and the secretion of IL-12 p70 and IFN- $\gamma$ to sensitize LLC1 tumor to chemotherapeutic drug cisplatin (CDDP) (46). Jurkat $\mathrm{T}$ cell line, a kind of CTLs, has been reported to resist tumor immune escape (55). Du et al. observed that Shenqi Fuzheng Injection (SFI) down-regulated the immunosuppressive cytokines IL-10, TGF- $\beta$ and VEGF in A375 cells in a concentration-dependent manner. Also, SFI enhanced the cytotoxic and migratory activities of Jurkat $\mathrm{T}$ cells in A375 melanoma to reprogram the melanoma microenvironment (50), which demonstrated that CTLs can mediate the antitumor effects of Chinese medicine compounds. In conclusion, 
TABLE 2 | Adaptive immune cells impacted by Chinese medicine compounds.

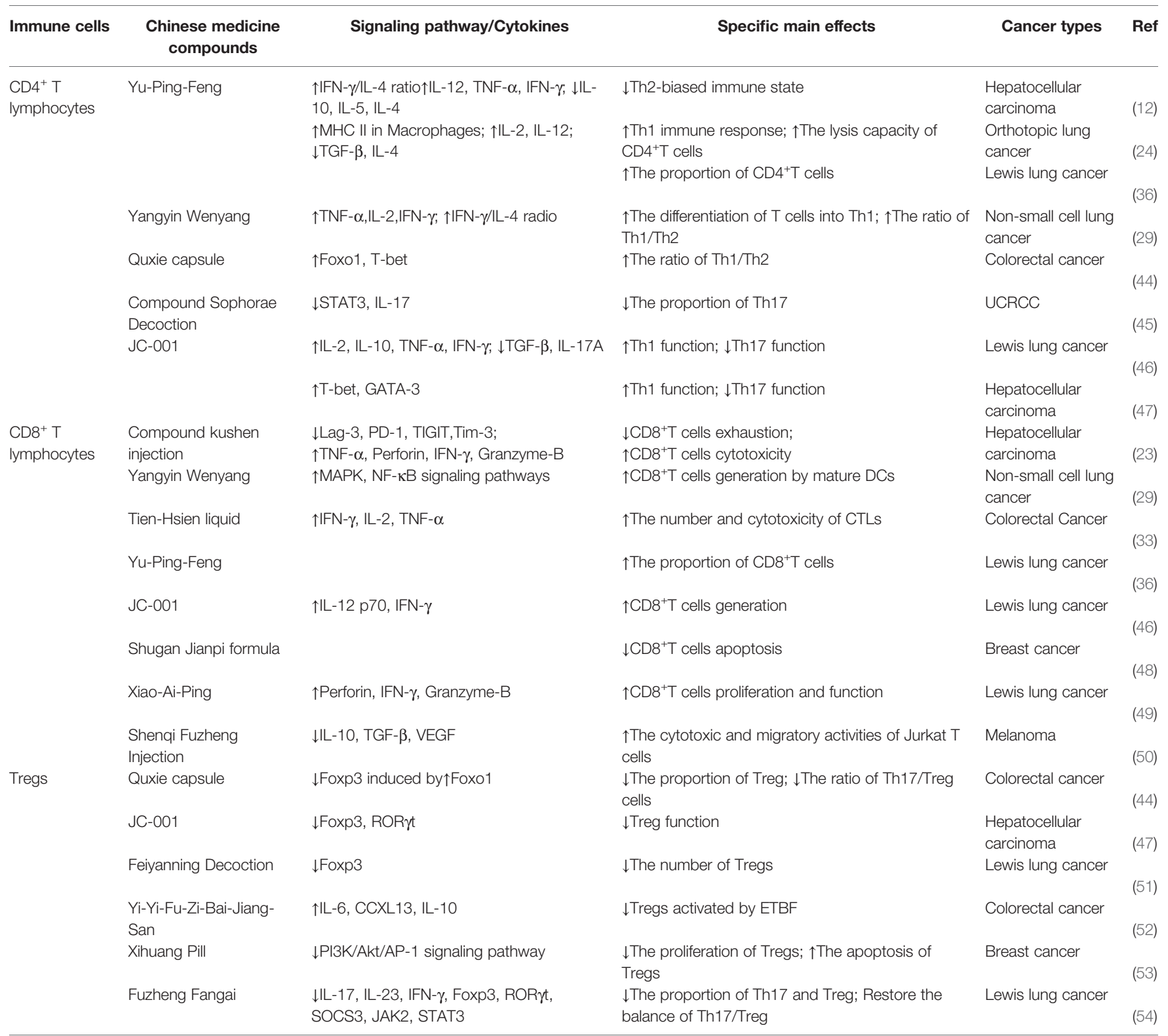

$\uparrow$, Up-regulated or enhanced; $\downarrow$, Down-regulated or inhibited.

the restoration and enhancement of the activity of $\mathrm{CD} 8^{+} \mathrm{T}$ cells are effective ways for Chinese medicine compounds to prevent cancer from deteriorating (Figure 1).

\section{Regulatory T Cells (Treg)}

Previous researches have shown the potential therapeutic effects of targeting Foxp $3^{+}$Tregs for cancer treatment (56). Guo et al. reported that Feiyanning Decoction significantly attenuated the amount of $\mathrm{CD} 4{ }^{+} \mathrm{CD} 25^{+}$regulatory $\mathrm{T}$ cells in spleen, thymus and tumor tissues in LLC-bearing mice. It decreased the mRNA level of Foxp3, enhanced tumor immune response, and slowed down the progression of lung cancer (51). Yi-Yi-Fu-Zi-Bai-Jiang-San (YYFZBJS), a classical Chinese medicine formula for gastrointestinal diseases, suppressed the number of
$\mathrm{CD} 4^{+} \mathrm{CD} 25^{+}$Foxp $^{+} \mathrm{T}$ cells. Although YYFZBJS did not directly affect CRC cells, it was able to down-regulate the proliferation of MC-38 cells through Enterotoxigenic Bacteroides fragilis (ETBF)-primed Tregs and preventing the advance of colon cancer (52). Moreover, Xihuang (XH) Pill reduced the proliferation of Tregs in the TME of breast cancer and induced the apoptosis of Tregs by suppressing PI3K/Akt/ AP-1 signaling pathway, thus reversing the immune escape to attenuate the tumor growth (53). The imbalance of Th17/Treg often occurs in autoimmune diseases and inflammation (57), which could be effectively ameliorated by Chinese medicine compounds. CSD restored the balance of Th17/Treg by decreasing the proportion of Th17, Treg and the related inflammatory factors to improve immune function (58). 
Inflammation is often closely associated with the advance of cancer, thereby adjusting Th17/Treg balance in cancer may be helpful to relieve tumor-mediated immunosuppression. On the one hand, QX down-regulated Foxo1-mediated Foxp3, reduced Tregs and recovered the balance of Th17/Treg, thus playing a therapeutic role in CRC (44). On the other hand, Liu et al. found that Fuzheng Fangai combined with cyclophosphamide (CTX) effectively decreased the proportion of Th17 and Treg in spleen and tumor tissues of LLC tumor-bearing mice and restored the ratio of Th17/Treg. Meanwhile, the secretion of IL-17, IL-23 and IFN- $\gamma$, the mRNA levels of Foxp3 and ROR $\gamma$ t as well as the protein levels of SOCS3, JAK2, and STAT3 were remarkably down-regulated by combined therapy compared to CTX alone (54). Summarily, Chinese medicine compounds are capable of suppressing tumor development through the inhibitory effects on Tregs (Table 2) (Figure 1).

\section{THE IMMUNOREGULATORY PATTERNS OF CHINESE MEDICINE COMPOUNDS IN CANCER THERAPY}

\section{Improve Tumor-Induced Inflammation}

Chronic inflammation serves as a risk factor for cancers. Tumorinduced inflammation accelerates the enrichment and activity of inflammatory cells in tumor tissue, thereby promoting tumor progression (59). Therefore, it may be feasible to treat cancers through improving inflammation to enhance immune function by Chinese medicine compounds (Figure 2). CRC was the third common cancer around the world, the morbidity of which ranked the second. Statistically, the incidence of CRC was higher in transition countries (1). Banxia Xiexin decoction (BXD) has been often applied to treat a variety of inflammatory diseases, including acute and chronic gastritis, oral ulcer, digestive ulcer, and so on. Yan et al. found that BXD enhanced the serum level of pro-inflammatory cytokines IL- $1 \beta$, TNF- $\alpha$ and IL-6 in colon cancer-bearing nude mice and improve inflammation (60). With the popularization of the hepatitis B vaccine, the total incidence and mortality of liver cancer have been declining in the past three decades, whereas primary liver cancer still continues. HCC is the most common primary liver cancer (1). Hepatic fibrosis is the most vital hazard factor of HCC (61). Yang et al. established chronic liver fibrosis models with $\mathrm{CCl}_{4}$ or MCD and found that CKI remarkably attenuated the infiltration of macrophages in the livers of these mice, downregulated the expression of TNF- $\alpha$ and IL- 6 , thus alleviating the inflammatory response induced by liver fibrosis. Furthermore, Smad7 was identified as a key target of CKI in the treatment of liver fibrosis. CKI up-regulated Smad7, inhibited TNF- $\beta R 1$, and suppressed TNF- $\beta /$ Smad signaling pathway to prevent the development of chronic liver fibrosis and HCC (62). NHE-06 exerted a strong anti-inflammatory activity on HCC through the suppression of NF- $\kappa \mathrm{B} / \mathrm{IL}-6 / \mathrm{STAT} 3$ signaling and TGF- $\alpha$ and PTSG2. Meanwhile, NHE-06 strengthened the anti-tumor immunity by increasing the infiltration of immune cells and the production of IFN- $\gamma$. However, the preventive and therapeutic efficacy against HCC could be merely realized in mice with an intact immune system (63). Breast cancer has become the most common malignant tumor in the world and one of the main causes of death in females (1). Ruyiping (RYP) has been reported as a traditional Chinese medicine formula for breast cancer metastasis. Ye et al. found that in breast cancer 4T1-bearing mice, RYP combined with Platycodon grandiflorum (RP) effectively decreased the content of inflammatory marker Fibrinogen and inflammation-associated cytokines IL-6 and $\mathrm{IL}-1 \beta$ in the lung tissue, improved the pulmonary inflammatory microenvironment resulted from breast cancer, and prevented the lung metastasis of breast cancer (64). According to Global Cancer Statistics 2020, lung cancer has become the most deadly malignant tumor in the world, whose morbidity ranked second among all cancers (1). Kejinyan decoction is an empirical prescription of traditional Chinese medicine for lung cancer clinically. Chen et al. reported that, after intervention with Kejinyan decoction, the inflammatory cytokines TNF- $\alpha$, IFN- $\gamma$, IL- 6 , IL- 4 and IL-13 were reduced and the survival of LLC tumor-bearing mice was prolonged (65). In short, Chinese medicine compounds could regulate the secretion of inflammatory cytokines to improve the inflammatory response in cancers.

\section{Enhance Immunity After Surgery or Chemotherapy}

Surgery and chemotherapy are the common treatments for cancers. However, side effects are always the major concern and limitation. Surgery tends to trigger inflammation, which leads to various unfavorable prognoses. Chemotherapy can damage the immune system even more. Therefore, Chinese medicine compounds combined with surgery or chemotherapy are typically used in clinical treatments to enhance efficacy and reduce side effects (Figure 2). Transcatheter arterial chemoembolization (TACE) has been identified as one of the therapies for HCC (66). Dai et al. reported DZP combined with TACE enhanced the immune functional indexes in serum and the number of $\mathrm{CD} 4^{+} / \mathrm{CD}^{+} \mathrm{T}$ cells and reduced VEGF, TGF- $\beta 1$ and MMP- 2 to ameliorate cancer metastasis and other adverse reactions after TACE (67). A clinical trial has demonstrated that San Huang decoction remarkably reduced the volume of exudate after breast cancer surgery and the expression of TNF- $\alpha$, IL-6, IL- 8 and C-reactive protein (CRP) to improve the inflammation. Some studies have illustrated that Chinese medicine compounds could not only enhance the effectiveness of chemotherapy $(23,46,48)$, but also alleviate the side effects of chemotherapy, including diarrhea, weight loss and other symptoms of hypoimmunity. PHY906 has been utilized for thousands of years to treat gastrointestinal diseases. In recent years, it has been developed as an adjuvant for cancer. Lam et al. found that PHY906 was capable of accelerating the infiltration of macrophages into tumors by inducing the up-regulation of macrophage cytokines hMCP1, dramatically promoted the conversion of macrophages to $\mathrm{M} 1$, and enhanced the anti-tumor effects of Sorafenib (68). In addition, Ciji-Hua'ai-Baosheng improved the immune function of $\mathrm{H}-22$ tumor-bearing mice after 
treatment with chemotherapy and alleviated CTX-induced colitis, manifested as elevated lymphocytes in spleen, augmented IL-2, IFN- $\gamma$ and TNF- $\alpha$, and the reduced IL- 6 in serum and tumor tissues (69). Similarly, Wu et al. found Gegen Qinlian decoction (GQT), a classic Chinese medicine compound widely applys to gastrointestinal inflammatory diseases, assisted the anti-tumor effects of irinotecan (CPT-11) on colon cancer. It reversed the abnormal enhancement of IL-1 $\beta$, COX-2, ICAM-1, and TNF- $\alpha$. Moreover, GQT relieved diarrhea in CPT-11-treated patients by restraining hCE2 (70). Tumor suppressor p53 has been considered to be able to regulate immunity. Inactivated p53 may influence the effects of BMCs and T cells (71). Wei et al. reported that Yangyin Fuzheng Decoction promoted the infiltration of inflammatory cells to improve anti-tumor immunity and restore the CDDP-mediated weight loss of mice (72). Thus, Chinese medicine compounds combined with conventional therapy are expected to be a promising treatment for patients suffering from malignant tumors.

\section{Block the Immune Checkpoints}

Immune checkpoints represent a series of molecules on immune cells, which are recognized as switches of immune functions. In the TME, immune checkpoints are often overexpressed or activated, leading to the paralysis of the immune system. PD-1 and its ligand 1 (PD-L1) are the pivotal immune checkpoints that promote immune escape and cancer advance. Targeting the PD-1/PD-L1 axis has been effective immunotherapy against cancer (73). The combined therapy of GQT and PD-1 blocker effectively accelerated the proliferation of $\mathrm{CD}^{+} \mathrm{T}$ cells and restored the T-cell function by up-regulating IL-2 and IFN- $\gamma$. Moreover, some Chinese medicine compounds themselves exerted blocking effects on immune checkpoints. Bu Fei Decoction adjusted the immunosuppressive TAMs in nonsmall cell lung cancer (NSCLC) and inhibited its tumorpromoting effect by down-regulating IL-10 and PD-L1 (74). Studies have found that the PI3K/Akt signaling pathway assisted the activation of PD-1/PD-L1 axis. Teng et al. summarized that the active ingredients of Huoxue Yiqi Recipe2 (HYR-2) targeted PD-L1 signaling pathway in the treatment of lung cancer. HYR-2 could turn M2 to M1 through downregulating PD-L1 that is closely associated with the blockage of PI3K/Akt signaling pathway (75). Xu et al. observed that Modified Bu-zhong-yi-qi decoction suppressed the expression of PD-L1 through blocking PI3K/Akt signaling pathway in gastric cancer, thereby up-regulating the ratio of $\mathrm{CD} 4^{+} / \mathrm{CD} 8^{+}$ $\mathrm{T}$ cells and the number of $\mathrm{CD} 8^{+} \mathrm{PD}-1^{+} \mathrm{T}$ cells as well as decreasing the proportion of $\mathrm{PD}-1^{+}$Tregs induced by chemotherapy, representing as a promising therapy for gastric cancer (76). PI3K $\gamma$ is an immune checkpoint for macrophages, which can switch the polarization of macrophages to reshape immune microenvironment and control immune suppression in cancers (77). Yuan et al. reported that Modified Jian-pi-yangzheng decoction down-regulated $\mathrm{PI} 3 \mathrm{~K} \gamma$ remarkably, promoted the secretion of TNF- $\alpha$ and IL- $1 \beta$ while decreased IL-10, accelerated the conversion of M2 to M1 and the differentiation of TAMs, thus prevented the progression and metastasis of gastric cancer (78) (Figure 2). Although immune checkpoint has gained great attention in tumor immunotherapy, the impacts of Chinese medicine compounds on immune checkpoints are not fully investigated.

\section{Other Potential Ways}

Recently, erythroid progenitor cell (EPC) has been known as the regulator in tumor immune response. In malignant tumors, EPCs did not differentiate into mature red blood cells and have inhibitory effects on tumor immunity (79). Li et al. reported that Danggui Buxue decoction reduced the abnormal accumulation of EPCs in melanoma and accelerated EPCs to differentiate into mature red blood cells, which led to relieving anemia, enhancing tumor immune response, and inhibiting the progression of melanoma (80). Estrogen/estrogen receptor (ER) has also been considered as a possible immunotherapy target for cancers (81). There are two forms of ER, ER $\alpha$ and ER $\beta$. When the increase in $\mathrm{ER} \alpha$ level leads to the imbalanced ratio of $\operatorname{ER} \alpha / \mathrm{ER} \beta$ or the mutation rate of ER $\alpha$ augments, it is more likely to induce breast cancer. XH formula has been used for the treatment of breast cancer since 1740, which suppressed the proliferation and activity of breast cancer cells in dose- and time-dependent manners. Hao et al. reported that $\mathrm{XH}$ formula bond to $\mathrm{ER} \alpha$ and HSP90, promoting the disintegration of ER $\alpha$, and blocking the transmission of ER $\alpha$ signaling. Through this anti-estrogenlike effect, $\mathrm{XH}$ formula inhibited the progression of breast tumors (82). Moreover, it was reported that Shugan Liangxue (SGLX) decoction suppressed the protein levels of ER $\alpha$ and 17 $\beta$-estradiol (E2) target genes, $\mathrm{c}-\mathrm{Myc}$ and $\mathrm{Bcl}-2$, in human breast cancer cells. It was speculated that SGLX exerted inhibitory effects on $\mathrm{ER}^{+}$ breast cancer cells selectively (83) (Figure 2).

\section{PERSPECTIVES}

There are various ways of Chinese medicine compounds to exert anti-tumor activity, including blocking cell cycle, inhibiting cell viability and proliferation, inducing cell apoptosis, preventing cell invasion and migration, and enhancing the sensitivity of tumor cells to chemotherapy drugs, etc. In recent years, immunotherapy has become the most promising field in cancer therapy. Regulating immune cells has been proved to be a powerful weapon against cancers and is increasingly applied clinically. Traditional Chinese medicine or its extracts have been reported to play a variety of roles in immune cells, thereby enhancing innate and adaptive immunity $(84,85)$. By the advantages of multi-components and multi-targets, Chinese medicine compounds have more comprehensive effects and mechanisms of regulating tumor immunity. Traditional Chinese medicine compound preparations, such as traditional Chinese medicine patent prescription and Chinese medicine compound injection, have been put into application clinically due to their anti-tumor and anti-inflammatory activity. The studies on mechanisms of Chinese medicine compounds enhancing tumor immunity mostly concentrated on the number and activity of immune cells in the TME, however, more in-depth and systematic researches are still needed and worth being further explored, including but not limited to: the targeted immune cell types which were regulated specifically by Chinese medicine compounds, the direct targets 
and pathways, either in immune cells or cancer cells, which mediated the immunotherapeutic effects of Chinese medicine compounds, the different immunomodulatory effects in distinct TME, the combined effect of immunoregulating Chinese medicine compounds with chemotherapy or immunotherapy drugs. Furthermore, the roles of various components in Chinese medicine compounds and their interplay or relationship in different symptoms is another important question. Nowadays, researchers try to depict formulas of traditional Chinese medicine compounds and optimize new formulations with more active ingredients and better therapeutic effects. Therefore, it is necessary to carry out more systematic researches on the immunoregulatory and pharmacological effect of TCM on cancer therapy and the underlying mechanisms to provide the more comprehensive theoretical basis for the clinical application of Chinese medicine compounds in cancer treatment.

\section{REFERENCES}

1. Sung H, Ferlay J, Siegel RL, Laversanne M, Soerjomataram I, Jemal A, et al. Global Cancer Statistics 2020: GLOBOCAN Estimates of Incidence and Mortality Worldwide for 36 Cancers in 185 Countries. CA Cancer J Clin (2021) 71(3):209-49. doi: 10.3322/caac.21660

2. Roma-Rodrigues $\mathrm{C}$, Mendes R, Baptista PV, Fernandes AR. Targeting Tumor Microenvironment for Cancer Therapy. Int J Mol Sci (2019) 20(4):840. doi: $10.3390 /$ ijms 20040840

3. Fan Y, Ma Z, Zhao L, Wang W, Gao M, Jia X, et al. Anti-Tumor Activities and Mechanisms of Traditional Chinese Medicines Formulas: A Review. BioMed Pharmacother (2020) 132:110820. doi: 10.1016/j.biopha.2020.110820

4. Dedong C, Huilin X, Anbing H, Ximing X, Wei G. The Effect of ShenQi FuZheng Injection in Combination With Chemotherapy Versus Chemotherapy Alone on the Improvement of Efficacy and Immune Function in Patients With Advanced Non-Small Cell Lung Cancer: A Meta-Analysis. PLoS One (2016) 11(3):e0152270. doi: 10.1371/ journal.pone. 0152270

5. Cao ZY, Chen XZ, Liao LM, Peng J, Hu HX, Liu ZZ, et al. Fuzheng Yiliu Granule Inhibits the Growth of Hepatocellular Cancer by Regulating Immune Function and Inducing Apoptosis In Vivo and In Vitro. Chin J Integr Med (2011) 17(9):691-7. doi: 10.1007/s11655-011-0847-3

6. Yang Q, Guo N, Zhou Y, Chen J, Wei Q, Han M. The Role of TumorAssociated Macrophages (TAMs) in Tumor Progression and Relevant Advance in Targeted Therapy. Acta Pharm Sin B (2020) 10(11):2156-70. doi: 10.1016/j.apsb.2020.04.004

7. Rhee I. Diverse Macrophages Polarization in Tumor Microenvironment. Arch Pharm Res (2016) 39(11):1588-96. doi: 10.1007/s12272-016-0820-y

8. Ma Y, Shurin GV, Peiyuan Z, Shurin MR. Dendritic Cells in the Cancer Microenvironment. J Cancer (2013) 4(1):36-44. doi: 10.7150/jca.5046

9. Tran Janco JM, Lamichhane P, Karyampudi L, Knutson KL. TumorInfiltrating Dendritic Cells in Cancer Pathogenesis. J Immunol (2015) 194 (7):2985-91. doi: 10.4049/jimmunol.1403134

10. Guillerey C. NK Cells in the Tumor Microenvironment. Adv Exp Med Biol (2020) 1273:69-90. doi: 10.1007/978-3-030-49270-0_4

11. Gabrilovich DI. Myeloid-Derived Suppressor Cells. Cancer Immunol Res (2017) 5(1):3-8. doi: 10.1158/2326-6066.Cir-16-0297

12. Yao F, Yuan Q, Song X, Zhou L, Liang G, Jiang G, et al. Yupingfeng Granule Improves Th2-Biased Immune State in Microenvironment of Hepatocellular Carcinoma Through TSLP-DC-OX40L Pathway. Evid Based Complement Alternat Med (2020) 2020:1263053. doi: 10.1155/2020/1263053

13. Basu A, Ramamoorthi G, Albert G, Gallen C, Beyer A, Snyder C, et al. Differentiation and Regulation of $\mathrm{T}(\mathrm{H})$ Cells: A Balancing Act for Cancer Immunotherapy. Front Immunol (2021) 12:669474. doi: 10.3389/ fimmu.2021.669474

14. Guéry L, Hugues S. Th17 Cell Plasticity and Functions in Cancer Immunity. BioMed Res Int (2015) 2015:314620. doi: 10.1155/2015/314620

\section{AUTHOR CONTRIBUTIONS}

FC drafted the manuscript. JL, HW and QB drafted and revised the manuscript. All authors contributed to the article and approved the submitted version.

\section{FUNDING}

This study was supported by grants from the National Natural Science Foundation of China (81630086, 82030099, and 81973078), National Key R\&D Program of China (2018YFC2000700), Shanghai Municipal Human Resources and Social Security Bureau (2018060), Shanghai Public Health System Construction Three-Year Action Plan (GWV-10.1-XK15), and Innovative Research Team of High-Level Local Universities in Shanghai.

15. Chang SH. T Helper 17 (Th17) Cells and Interleukin-17 (IL-17) in Cancer. Arch Pharm Res (2019) 42(7):549-59. doi: 10.1007/s12272-019-01146-9

16. He QF, Xu Y, Li J, Huang ZM, Li XH, Wang X. CD8+ T-Cell Exhaustion in Cancer: Mechanisms and New Area for Cancer Immunotherapy. Brief Funct Genomics (2019) 18(2):99-106. doi: 10.1093/bfgp/ely006

17. Takeuchi $\mathrm{Y}$, Nishikawa $\mathrm{H}$. Roles of Regulatory $\mathrm{T}$ Cells in Cancer Immunity. Int Immunol (2016) 28(8):401-9. doi: 10.1093/intimm/dxw025

18. Zhou J, Li X, Wu X, Zhang T, Zhu Q, Wang X, et al. Exosomes Released From Tumor-Associated Macrophages Transfer miRNAs That Induce a Treg/Th17 Cell Imbalance in Epithelial Ovarian Cancer. Cancer Immunol Res (2018) 6 (12):1578-92. doi: 10.1158/2326-6066.Cir-17-0479

19. Li X, Liu R, Su X, Pan Y, Han X, Shao C, et al. Harnessing Tumor-Associated Macrophages as Aids for Cancer Immunotherapy. Mol Cancer (2019) 18 (1):177. doi: 10.1186/s12943-019-1102-3

20. Orecchioni M, Ghosheh Y, Pramod AB, Ley K. Macrophage Polarization: Different Gene Signatures in M1(LPS+) vs. Classically and M2(LPS-) vs. Alternatively Activated Macrophages. Front Immunol (2019) 10:1084. doi: 10.3389/fimmu.2019.01084

21. Zhou JY, Chen M, Wu CE, Zhuang YW, Chen YG, Liu SL. The Modified SiJun-Zi Decoction Attenuates Colon Cancer Liver Metastasis by Increasing Macrophage Cells. BMC Complement Altern Med (2019) 19(1):86. doi: 10.1186/s12906-019-2498-4

22. Ma WP, Hu SM, Xu YL, Li HH, Ma XQ, Wei BH, et al. Haimufang Decoction, a Chinese Medicine Formula for Lung Cancer, Arrests Cell Cycle, Stimulates Apoptosis in NCI-H1975 Cells, and Induces M1 Polarization in RAW 264.7 Macrophage Cells. BMC Complement Med Ther (2020) 20(1):243. doi: 10.1186/s12906-020-03031-1

23. Yang Y, Sun M, Yao W, Wang F, Li X, Wang W, et al. Compound Kushen Injection Relieves Tumor-Associated Macrophage-Mediated Immunosuppression Through TNFR1 and Sensitizes Hepatocellular Carcinoma to Sorafenib. J Immunother Cancer (2020) 8(1):e000317. doi: 10.1136/jitc-2019-000317

24. Wang L, Wu W, Zhu X, Ng W, Gong C, Yao C, et al. The Ancient Chinese Decoction Yu-Ping-Feng Suppresses Orthotopic Lewis Lung Cancer Tumor Growth Through Increasing M1 Macrophage Polarization and CD4(+) T Cell Cytotoxicity. Front Pharmacol (2019) 10:1333. doi: 10.3389/fphar.2019.01333

25. Zheng Y, Wang N, Wang S, Yang B, Situ H, Zhong L, et al. XIAOPI Formula Inhibits the Pre-Metastatic Niche Formation in Breast Cancer via Suppressing TAMs/CXCL1 Signaling. Cell Commun Signal (2020) 18(1):48. doi: 10.1186/ s12964-020-0520-6

26. Deshmane SL, Kremlev S, Amini S, Sawaya BE. Monocyte Chemoattractant Protein-1 (MCP-1): An Overview. J Interferon Cytokine Res (2009) 29(6):31326. doi: $10.1089 /$ jir.2008.0027

27. Chen C, Yao X, Xu Y, Zhang Q, Wang H, Zhao L, et al. Dahuang Zhechong Pill Suppresses Colorectal Cancer Liver Metastasis via Ameliorating Exosomal CCL2 Primed Pre-Metastatic Niche. J Ethnopharmacol (2019) 238:111878. doi: $10.1016 /$ j.jep.2019.111878 
28. Wu J, Zhang XX, Zou X, Wang M, Wang HX, Wang YH, et al. The Effect of Jianpi Yangzheng Xiaozheng Decoction and its Components on Gastric Cancer. J Ethnopharmacol (2019) 235:56-64. doi: 10.1016/j.jep.2019.02.003

29. Zhao B, Hui X, Jiao L, Bi L, Wang L, Huang P, et al. A TCM Formula YYWY Inhibits Tumor Growth in Non-Small Cell Lung Cancer and Enhances Immune-Response Through Facilitating the Maturation of Dendritic Cells. Front Pharmacol (2020) 11:798. doi: 10.3389/fphar.2020.00798

30. Yang Y, Ma Y, Wang Z, Wang L, Zhao Y, Hui Y, et al. Compound Kushen Injection Promoted the Killing Effect of Cytokine-Induced Killer Cells Which Was Activated by Dendritic-Colon Cancer Stem Cell Fusion Cells on Colon Cancer Stem Cells. J Biomater Tissue Eng (2020) 10(7):957-65. doi: 10.1166/ jbt.2020.2362

31. Luo Y, Wu J, Zhu X, Gong C, Yao C, Ni Z, et al. NK Cell-Dependent Growth Inhibition of Lewis Lung Cancer by Yu-Ping-Feng, an Ancient Chinese Herbal Formula. Mediators Inflamm (2016) 2016:3541283. doi: 10.1155/2016/ 3541283

32. Que ZJ, Yao JL, Zhou ZY, Yu P, Luo B, Li HG, et al. Jinfukang Inhibits Lung Cancer Metastasis by Upregulating CX3CL1 to Recruit NK Cells to Kill CTCs. J Ethnopharmacol (2021) 275:114175. doi: 10.1016/j.jep.2021.114175

33. Yang PM, Du JL, Wang GN, Chia JS, Hsu WB, Pu PC, et al. The Chinese Herbal Mixture Tien-Hsien Liquid Augments the Anticancer Immunity in Tumor Cell-Vaccinated Mice. Integr Cancer Ther (2017) 16(3):319-28. doi: $10.1177 / 1534735416651492$

34. Hong-Fen L, Waisman T, Maimon Y, Shakhar K, Rosenne E, Ben-Eliyahu S. The Effects of a Chinese Herb Formula, Anti-Cancer Number One (ACNO), on NK Cell Activity and Tumor Metastasis in Rats. Int Immunopharmacol (2001) 1(11):1947-56. doi: 10.1016/s1567-5769(01)00120-5

35. Mao D, Feng L, Gong H. The Antitumor and Immunomodulatory Effect of Yanghe Decoction in Breast Cancer Is Related to the Modulation of the JAK/ STAT Signaling Pathway. Evid Based Complement Alternat Med (2018) 2018:8460526. doi: 10.1155/2018/8460526

36. Wang Y, Sun N, Luo Y, Fang Z, Fang Y, Tian J, et al. Yu-Ping-Feng Formula Exerts Antilung Cancer Effects by Remodeling the Tumor Microenvironment Through Regulating Myeloid-Derived Suppressor Cells. Evid Based Complement Alternat Med (2021) 2021:6624461. doi: 10.1155/2021/6624461

37. Xie Y, Zhang Y, Wei X, Zhou C, Huang Y, Zhu X, et al. Jianpi Huayu Decoction Attenuates the Immunosuppressive Status of H(22) Hepatocellular Carcinoma-Bearing Mice: By Targeting Myeloid-Derived Suppressor Cells. Front Pharmacol (2020) 11:16. doi: 10.3389/fphar.2020.00016

38. Tian S, Song X, Wang Y, Wang X, Mou Y, Chen Q, et al. Chinese Herbal Medicine Baoyuan Jiedu Decoction Inhibits the Accumulation of Myeloid Derived Suppressor Cells in Pre-Metastatic Niche of Lung via TGF- $\beta / C C L 9$ Pathway. BioMed Pharmacother (2020) 129:110380. doi: 10.1016/ j.biopha.2020.110380

39. Xu ZH, Zhu YZ, Su L, Tang XY, Yao C, Jiao XN, et al. Ze-Qi-Tang Formula Induces Granulocytic Myeloid-Derived Suppressor Cell Apoptosis via STAT3/S100A9/Bcl-2/Caspase-3 Signaling to Prolong the Survival of Mice With Orthotopic Lung Cancer. Mediators Inflamm (2021) 2021:8856326. doi: $10.1155 / 2021 / 8856326$

40. Wei H, Guo C, Zhu R, Zhang C, Han N, Liu R, et al. Shuangshen Granules Attenuate Lung Metastasis by Modulating Bone Marrow Differentiation Through mTOR Signalling Inhibition. J Ethnopharmacol (2020) 281:113305. doi: 10.1016/j.jep.2020.113305

41. Guéry JC, Ria F, Galbiati F, Adorini L. Antigen Presentation and IL-12 Production by Dendritic Cells In Vivo. Adv Exp Med Biol (1997) 417:317-21. doi: 10.1007/978-1-4757-9966-8_52

42. Ito T, Wang YH, Duramad O, Hori T, Delespesse GJ, Watanabe N, et al. TSLP-Activated Dendritic Cells Induce an Inflammatory T Helper Type 2 Cell Response Through OX40 Ligand. J Exp Med (2005) 202(9):1213-23. doi: $10.1084 /$ jem. 20051135

43. Shimasaki N, Jain A, Campana D. NK Cells for Cancer Immunotherapy. Nat Rev Drug Discov (2020) 19(3):200-18. doi: 10.1038/s41573-019-0052-1

44. Chen D, Yang Y, Yang P. Quxie Capsule Inhibits Colon Tumor Growth Partially Through Foxol-Mediated Apoptosis and Immune Modulation. Integr Cancer Ther (2019) 18:1534735419846377. doi: 10.1177/ 1534735419846377

45. Deng S, Tang Q, Duan X, Fan H, Zhang L, Zhu X, et al. Uncovering the Anticancer Mechanism of Compound Sophorae Decoction Against Ulcerative
Colitis-Related Colorectal Cancer in Mice. Evid Based Complement Alternat Med (2019) 2019:8128170. doi: 10.1155/2019/8128170

46. Chuang MH, Jan MS, Chang JT, Lu FJ. The Chinese Medicine JC-001 Enhances the Chemosensitivity of Lewis Lung Tumors to Cisplatin by Modulating the Immune Response. BMC Complement Altern Med (2017) 17(1):210. doi: 10.1186/s12906-017-1728-x

47. Chuang MH, Chang JT, Hsu LJ, Jan MS, Lu FJ. Antitumor Activity of the Chinese Medicine JC-001 Is Mediated by Immunomodulation in a Murine Model of Hepatocellular Carcinoma. Integr Cancer Ther (2017) 16(4):516-25. doi: 10.1177/1534735416664173

48. Lu YT, Li J, Qi X, Pei YX, Shi WG, Lin HS. Effects of Shugan Jianpi Formula () on Myeloid-Derived Suppression Cells-Mediated Depression Breast Cancer Mice. Chin J Integr Med (2017) 23(6):453-60. doi: 10.1007/s11655-016-2734-4

49. Li W, Yang Y, Ouyang Z, Zhang Q, Wang L, Tao F, et al. Xiao-Ai-Ping, a TCM Injection, Enhances the Antigrowth Effects of Cisplatin on Lewis Lung Cancer Cells Through Promoting the Infiltration and Function of CD8(+) T Lymphocytes. Evid Based Complement Alternat Med (2013) 2013:879512. doi: $10.1155 / 2013 / 879512$

50. Du J, Cheng BC, Fu XQ, Su T, Li T, Guo H, et al. In Vitro Assays Suggest Shenqi Fuzheng Injection has the Potential to Alter Melanoma Immune Microenvironment. J Ethnopharmacol (2016) 194:15-9. doi: 10.1016/ j.jep.2016.08.038

51. Guo J, Wang JY, Zheng Z, Wang Q, Dong CS. [Effects of Chinese Herbal Medicine Feiyanning Decoction on the Ratio of CD4+CD25+ Regulatory T Cells and Expression of Transcription Factor Foxp3 in Mice Bearing Lewis Lung Carcinoma]. Zhong Xi Yi Jie He Xue Bao (2012) 10(5):584-90. doi: 10.3736/jcim20120515

52. Sui H, Zhang L, Gu K, Chai N, Ji Q, Zhou L, et al. YYFZBJS Ameliorates Colorectal Cancer Progression in $\mathrm{Apc}(\mathrm{Min} /+)$ Mice by Remodeling Gut Microbiota and Inhibiting Regulatory T-Cell Generation. Cell Commun Signal (2020) 18(1):113. doi: 10.1186/s12964-020-00596-9

53. Su L, Jiang Y, Xu Y, Li X, Gao W, Xu C, et al. Xihuang Pill Promotes Apoptosis of Treg Cells in the Tumor Microenvironment in 4T1 Mouse Breast Cancer by Upregulating MEKK1/SEK1/JNK1/AP-1 Pathway. BioMed Pharmacother (2018) 102:1111-9. doi: 10.1016/j.biopha.2018.03.063

54. Liu S, Wang XM, Yang GW. Action Mechanism of Fuzheng Fangai Pill Combined With Cyclophosphamide on Tumor Metastasis and Growth. Evid Based Complement Alternat Med (2014) 2014:494528. doi: 10.1155/2014/494528

55. Kassouf N, Thornhill MH. Oral Cancer Cell Lines can Use Multiple Ligands, Including Fas-L, TRAIL and TNF-Alpha, to Induce Apoptosis in Jurkat T Cells: Possible Mechanisms for Immune Escape by Head and Neck Cancers. Oral Oncol (2008) 44(7):672-82. doi: 10.1016/j.oraloncology.2007.08.013

56. Saleh R, Elkord E. FoxP3(+) T Regulatory Cells in Cancer: Prognostic Biomarkers and Therapeutic Targets. Cancer Lett (2020) 490:174-85. doi: $10.1016 /$ j.canlet.2020.07.022

57. Noack M, Miossec P. Th17 and Regulatory T Cell Balance in Autoimmune and Inflammatory Diseases. Autoimmun Rev (2014) 13(6):668-77. doi: 10.1016/j.autrev.2013.12.004

58. Xu M, Duan XY, Chen QY, Fan H, Hong ZC, Deng SJ, et al. Effect of Compound Sophorae Decoction on Dextran Sodium Sulfate (DSS)-Induced Colitis in Mice by Regulating Th17/Treg Cell Balance. BioMed Pharmacother (2019) 109:2396-408. doi: 10.1016/j.biopha.2018.11.087

59. Diakos CI, Charles KA, McMillan DC, Clarke SJ. Cancer-Related Inflammation and Treatment Effectiveness. Lancet Oncol (2014) 15(11): e493-503. doi: 10.1016/s1470-2045(14)70263-3

60. Yan S, Yue Y, Wang J, Li W, Sun M, Zeng L, et al. Banxia Xiexin Decoction, a Traditional Chinese Medicine, Alleviates Colon Cancer in Nude Mice. Ann Transl Med (2019) 7(16):375. doi: 10.21037/atm.2019.07.26

61. Shankaraiah RC, Callegari E, Guerriero P, Rimessi A, Pinton P, Gramantieri L, et al. Metformin Prevents Liver Tumourigenesis by Attenuating Fibrosis in a Transgenic Mouse Model of Hepatocellular Carcinoma. Oncogene (2019) 38 (45):7035-45. doi: 10.1038/s41388-019-0942-Z

62. Yang Y, Sun M, Li W, Liu C, Jiang Z, Gu P, et al. Rebalancing TGF- $\beta /$ Smad7 Signaling via Compound Kushen Injection in Hepatic Stellate Cells Protects Against Liver Fibrosis and Hepatocarcinogenesis. Clin Transl Med (2021) 11: e410. doi: $10.1002 / \mathrm{ctm} 2.410$

63. Lu X, Wo G, Li B, Xu C, Wu J, Jiang C, et al. The Anti-Inflammatory NHE-06 Restores Antitumor Immunity by Targeting NF-Kb/LL-6/STAT3 Signaling in 
Hepatocellular Carcinoma. BioMed Pharmacother (2018) 102:420-7. doi: 10.1016/j.biopha.2018.03.099

64. Ye Y, Pei L, Wu C, Liu S. Protective Effect of Traditional Chinese Medicine Formula RP on Lung Microenvironment in Pre-Metastasis Stage of Breast Cancer. Integr Cancer Ther (2019) 18:1534735419876341. doi: 10.1177/ 1534735419876341

65. Chen M, Hu C, Gao Q, Li L, Cheng Z, Li Q, et al. Study on Metastasis Inhibition of Kejinyan Decoction on Lung Cancer by Affecting Tumor Microenvironment. Cancer Cell Int (2020) 20:451. doi: 10.1186/s12935-02001540-0

66. Liu J, Yi J. Relationship Between the Changes of VEGF Level and Dendritic Cells in Peripheral Blood of Patients With Hepatocellular Carcinoma After Transcatheter Arterial Chemoembolization. J Huazhong Univ Sci Technolog Med Sci (2007) 27(1):58-60. doi: 10.1007/s11596-007-0117-y

67. Dai CM, Jin S, Zhang JZ. Effect of Dahuang Zhechong Pills Combined With TACE on VEGF, MMP-2, TGF- $\beta 1$ and Immune Function of Patients With Primary Liver Cancer (Blood Stasis and Collaterals Blocking Type). Zhongguo Zhong Yao Za Zhi (2021) 46(3):722-9. doi: 10.19540/j.cnki.cjcmm. 20200716.501

68. Lam W, Jiang Z, Guan F, Huang X, Hu R, Wang J, et al. PHY906(KD018), an Adjuvant Based on a 1800-Year-Old Chinese Medicine, Enhanced the AntiTumor Activity of Sorafenib by Changing the Tumor Microenvironment. Sci Rep (2015) 5:9384. doi: 10.1038/srep09384

69. Xi S, Fu B, Loy G, Minuk GY, Peng Y, Qiu Y, et al. The Effects of Ciji-Hua'aiBaosheng on Immune Function of Mice With H(22) Hepatocellular Carcinoma Receiving Chemotherapy. BioMed Pharmacother (2018) 101:898-909. doi: 10.1016/j.biopha.2018.03.027

70. Wu Y, Wang D, Yang X, Fu C, Zou L, Zhang J. Traditional Chinese Medicine Gegen Qinlian Decoction Ameliorates Irinotecan Chemotherapy-Induced Gut Toxicity in Mice. BioMed Pharmacother (2019) 109:2252-61. doi: 10.1016/j.biopha.2018.11.095

71. Blagih J, Buck MD, Vousden KH. P53, Cancer and the Immune Response. J Cell Sci (2020) 133(5):jcs237453. doi: 10.1242/jcs.237453

72. Wei D, Wang L, Chen Y, Yin G, Jiang M, Liu R, et al. Yangyin Fuzheng Decoction Enhances Anti-Tumor Efficacy of Cisplatin on Lung Cancer. J Cancer (2018) 9(9):1568-74. doi: 10.7150/jca.24525

73. Ai L, Xu A, Xu J. Roles of PD-1/PD-L1 Pathway: Signaling, Cancer, and Beyond. Adv Exp Med Biol (2020) 1248:33-59. doi: 10.1007/978-981-15-3266-5_3

74. Pang L, Han S, Jiao Y, Jiang S, He X, Li P. Bu Fei Decoction Attenuates the Tumor Associated Macrophage Stimulated Proliferation, Migration, Invasion and Immunosuppression of non-Small Cell Lung Cancer, Partially via IL-10 and PD-L1 Regulation. Int J Oncol (2017) 51(1):25-38. doi: 10.3892/ ijo.2017.4014

75. Teng L, Wang K, Chen W, Wang YS, Bi L. HYR-2 Plays an Anti-Lung Cancer Role by Regulating PD-L1 and Akkermansia Muciniphila. Pharmacol Res (2020) 160:105086. doi: 10.1016/j.phrs.2020.105086

76. Xu R, Wu J, Zhang X, Zou X, Li C, Wang H, et al. Modified Bu-Zhong-Yi-Qi Decoction Synergies With 5 Fluorouracile to Inhibits Gastric Cancer Progress via PD-1/PD- L1-Dependent T Cell Immunization. Pharmacol Res (2020) 152:104623. doi: 10.1016/j.phrs.2019.104623

77. Kaneda MM, Messer KS, Ralainirina N, Li H, Leem CJ, Gorjestani S, et al. Pi3k $\gamma$ Is a Molecular Switch That Controls Immune Suppression. Nature (2016) 539(7629):437-42. doi: 10.1038/nature19834

78. Yuan M, Zou X, Liu S, Xu X, Wang H, Zhu M, et al. Modified Jian-Pi-YangZheng Decoction Inhibits Gastric Cancer Progression via the Macrophage Immune Checkpoint PI3K $\gamma$. BioMed Pharmacother (2020) 129:110440. doi: 10.1016/j.biopha.2020.110440

79. Grzywa TM, Justyniarska M, Nowis D, Golab J. Tumor Immune Evasion Induced by Dysregulation of Erythroid Progenitor Cells Development. Cancers (Basel) (2021) 13(4):870. doi: 10.3390/cancers13040870

80. Li C, Zhu F, Xu C, Xiao P, Wen J, Zhang X, et al. Dangguibuxue Decoction Abolishes Abnormal Accumulation of Erythroid Progenitor Cells Induced by Melanoma. J Ethnopharmacol (2019) 242:112035. doi: 10.1016/j.jep.2019.112035

81. Wang T, Jin J, Qian C, Lou J, Lin J, Xu A, et al. Estrogen/ER in Anti-Tumor Immunity Regulation to Tumor Cell and Tumor Microenvironment. Cancer Cell Int (2021) 21(1):295. doi: 10.1186/s12935-021-02003-w

82. Hao J, Jin Z, Zhu H, Liu X, Mao Y, Yang X, et al. Antiestrogenic Activity of the Xi-Huang Formula for Breast Cancer by Targeting the Estrogen Receptor $\alpha$. Cell Physiol Biochem (2018) 47(6):2199-215. doi: 10.1159/000491533

83. Zhou N, Han SY, Chen YZ, Zhou F, Zheng WX, Li PP. Shugan Liangxue Decoction () Down-Regulates Estrogen Receptor $\alpha$ Expression in Breast Cancer Cells. Chin J Integr Med (2018) 24(7):518-24. doi: 10.1007/s11655015-2123-4

84. Wang S, Long S, Deng Z, Wu W. Positive Role of Chinese Herbal Medicine in Cancer Immune Regulation. Am J Chin Med (2020) 48(7):1577-92. doi: 10.1142/s0192415x20500780

85. Wang Y, Zhang Q, Chen Y, Liang CL, Liu H, Qiu F, et al. Antitumor Effects of Immunity-Enhancing Traditional Chinese Medicine. BioMed Pharmacother (2020) 121:109570. doi: 10.1016/j.biopha.2019.109570

Conflict of Interest: The authors declare that the research was conducted in the absence of any commercial or financial relationships that could be construed as a potential conflict of interest.

Publisher's Note: All claims expressed in this article are solely those of the authors and do not necessarily represent those of their affiliated organizations, or those of the publisher, the editors and the reviewers. Any product that may be evaluated in this article, or claim that may be made by its manufacturer, is not guaranteed or endorsed by the publisher.

Copyright $\odot 2021$ Chen, Li, Wang and Ba. This is an open-access article distributed under the terms of the Creative Commons Attribution License (CC BY). The use, distribution or reproduction in other forums is permitted, provided the original author(s) and the copyright owner(s) are credited and that the original publication in this journal is cited, in accordance with accepted academic practice. No use, distribution or reproduction is permitted which does not comply with these terms. 


\section{GLOSSARY}

\begin{tabular}{|c|c|}
\hline ACNO & Anti-cancer No.1 \\
\hline Akt & Protein kinase B \\
\hline AP-1 & Activator protein-1 \\
\hline APCs & Antigen presenting cells \\
\hline Arg-1 & Arginase-1 \\
\hline Bax & Bcl2-Associated X \\
\hline Bcl-2 & B-cell lymphoma-2 \\
\hline BMCs & Bone marrow cells \\
\hline BMDCs & Bone marrow dendritic cells \\
\hline BXD & Banxia Xiexin decoction \\
\hline BYJD & Baoyuan Jiedu decoction \\
\hline CCL2/CCR2 & $\begin{array}{l}\text { Chemokine (C-C motif) ligand } 2 / \mathrm{C}-\mathrm{C} \text { chemokine receptor } \\
\text { type } 2\end{array}$ \\
\hline $\mathrm{CCl}_{4}$ & Carbon tetrachloride \\
\hline CCL9 & Chemokine (C-C motif) ligand 9 \\
\hline CDDP & Cisplatin \\
\hline ClK cells & Cytokines induce killer cells \\
\hline CKI & Compound kushen injection \\
\hline $\mathrm{COX}-2$ & Cyclooxygenase-2 \\
\hline CPT-11 & irinotecan \\
\hline CRC & Colorectal cancer \\
\hline CRP & C-reactive protein \\
\hline CSCs & Cancer stem cells \\
\hline CSD & Compound Sophorae Decoction \\
\hline CTCs & Circulating tumor cells \\
\hline CTL/Tc & Cytotoxic T cell \\
\hline CTX & Cyclophosphamide \\
\hline CXCL1 & Chemokine (C-X-C motif) Ligand 1 \\
\hline CX3CL1 & Chemokine (C-X3-C motif) Ligand 1 \\
\hline DCs & Dendritic cells \\
\hline DC-cCSC & Fusion cells of dendritic colon cancer stem cells \\
\hline \multicolumn{2}{|r|}{ 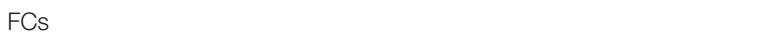 } \\
\hline DZP & Dahuang Zhechong Pills \\
\hline E2 & Estradiol \\
\hline EPC & Erythroid progenitor cell \\
\hline ER & Estrogen receptor \\
\hline ERK & Extracellular regulated protein kinases \\
\hline ETBF & enterotoxigenic bacteroides fragilis \\
\hline Fas/FasL & Factor associated suicide/Fas Ligand \\
\hline FCs & Fusion cells \\
\hline FN & Fibronectin \\
\hline Foxo1 & Forkhead box $\mathrm{O} 1$ \\
\hline Foxp3 & Forkhead box p3 \\
\hline GEM & gemcitabine \\
\hline GM-CSF & Granulocyte-macrophage colony stimulating factor \\
\hline GQT & Gegen Qinlian decoction \\
\hline $\mathrm{HCC}$ & Hepatocellular carcinoma \\
\hline hCE2 & human carboxylesterase 2 \\
\hline hMCP1 & Human monocyte chemoattractant protein-1 \\
\hline HMF & Haimufang \\
\hline HSPCs & Hematopoietic stem-progenitor cells \\
\hline HSP90 & Heat shock protein 90 \\
\hline HYR-2 & Huoxue Yiqi Recipe-2 \\
\hline ICAM-1 & Intercellular adhesion molecule-1 \\
\hline IFN- $\gamma / \mathrm{INF}-\gamma$ & Interferon-gamma \\
\hline $\mathrm{IL}$ & Interleukin \\
\hline
\end{tabular}

(Continued)
Continued

\begin{tabular}{|c|c|}
\hline iNOS & Inducible nitric oxide synthase \\
\hline JAK & Janus Kinase \\
\hline $\mathrm{JHD}$ & Jianpi Huayu Decoction \\
\hline JPYZ & Jianpi Yangzheng \\
\hline Lag-3 & Lymphocyte-activation gene 3 \\
\hline LLC & Lewis lung cancer \\
\hline MAPK & Mitogen-activated protein kinase \\
\hline MCD & Methionine-choline deficient \\
\hline MDSCs & Myeloid-derived suppressor cells \\
\hline MEK & Mitogen-activated protein kinase kinase \\
\hline $\mathrm{MHC}$ & Major histocompatibility complex \\
\hline MMP & Matrix metalloproteinase \\
\hline mTOR & Mechanistic target of rapamycin \\
\hline Myc & Myelocytomatosis oncogene \\
\hline $\mathrm{NF}-\kappa \mathrm{B}$ & nuclear factor kappa-B \\
\hline NK cells & Natural killer cells \\
\hline NKT cells & Nature killer T cells \\
\hline NO & nitric oxide \\
\hline NSCLC & Non-small cell lung cancer \\
\hline PARP & Poly ADP-ribose polymerase \\
\hline PD-1/PD-L1 & $\begin{array}{l}\text { Programmed cell death protein 1/programmed cell death ligand } \\
1\end{array}$ \\
\hline PI3K & phosphatidylinositol 3 kinase \\
\hline PMN & Pre-metastatic niche \\
\hline PTSG2 & Prostaglandin-endoperoxide synthase 2 \\
\hline QX & Quxie capsule \\
\hline ROS & Reactive oxygen species \\
\hline RORyt & Retinoid-related orphan receptor-gammat \\
\hline $\mathrm{RP}$ & RYP with Platycodon grandiflorum \\
\hline RYP & Ruyiping \\
\hline S6K1 & Ribsosmal protein S6 kinase 1 \\
\hline SFI & ShenQi FuZheng injection \\
\hline SGLX & Shugan Liangxue \\
\hline Smad & Small mothers against decapentaplegic \\
\hline SOCS3 & Suppressor of cytokine signaling 3 \\
\hline STAT & Signal transducer and activator of transcription \\
\hline T cell & T lymphocyte \\
\hline TACE & Transcatheter arterial chemoembolization \\
\hline TAMs & Tumor associated macrophages \\
\hline TCM & Traditional Chinese medicine \\
\hline TGF & Transforming growth factor \\
\hline Th & Helper T lymphocytes \\
\hline Tho & naïve T lymphocytes \\
\hline THL & Tien-Hsien liquid \\
\hline TIGIT & T-cell immunoreceptor with Ig and ITIM domains \\
\hline Tim-3 & T-cell immunoglobulin and mucin-domain containing-3 \\
\hline TME & Tumor microenvironment \\
\hline TNF & Tumor necrosis factor \\
\hline TNFR1 & Tumor necrosis factor receptor superfamily member 1 \\
\hline Treg & Regulatory $\mathrm{T}$ cell \\
\hline TSLP & Thymic stromal lymphopoietin \\
\hline UCRCC & Ulcerative Colitis-Related Colorectal Cancer \\
\hline VEGF & Vascular endothelial growth factor \\
\hline $\mathrm{XP}$ & XIAOPI \\
\hline $\mathrm{XH}$ & Xi Huang \\
\hline YHD & Yanghe Decoction \\
\hline YPF & Yu-ping-feng \\
\hline YYFZBJS & Yi-Yi-Fu-Zi-Bai-Jiang-San \\
\hline YYWY & Yangyin Wenyang \\
\hline ZQT & Ze-Qi-Tang \\
\hline
\end{tabular}

